percentage score. Finally the scores for each sub-criteria are added up to the total score of each country.

Result(s)* Belgium, Denmark, Ireland and the UK are the policy champions and lead the Atlas with excellent policies on primary secondary prevention and providing evidence based information to citizens.

Romania, Bulgaria and Slovakia worst performing countries in the EU (no funding for vaccines, vaccine only available to girls, poorly organised screening programmes, absence of reliable online information). In terms of geographical Europe, Belarus and Azerbaijan score the worse, as there is literally no information about the HPV prevention to be found and policies on primary or secondary prevention are non-existent.

Conclusion* The situation in Europe is very unequal. There is a clear divide between northern Europe, Southern Europe and Eastern Europe. While vaccine exists and screenings technologies are available - today the access is very dependent on where you live. This leads to high incidence and mortality which could be avoided should proper policies be put in place.

Links ATLAS:

EPF:

\section{RAISING ADEQUATE VAGINAL MARGINS DURING COLPOTOMY FOR CERVICAL CANCER}

T Shylasree*, B Dash, P Poddar. Tata Memorial Hospital, Gynecological Oncology, Mumbai, India

\subsection{6/ijgc-2021-ESGO.54}

Introduction/Background* Adequate surgical vaginal margins are pre-requisite for improving oncological outcomes in cervical cancer and precancer. Raising the margins through vaginal route helps in visualizing and measuring the vaginal and is more accurate than performing colpotomy from a open abdominal/Minimal access route.

Methodology Following completion of ligation of uterine vessels with or without adequate parametrium depending on the indication for radicality (abdominal/minimal access route,/ schuatas vaginal hysterectomy), surgeon moves to the bottom end of the patient. Cervix is visualised and held with volsellum. Circumferential vaginal margin which needs to be removed is marked with cautery. Vaginal mucosa is infiltrated with saline with or without adrenaline. With the help of electrical diathermy vaginal margins are raised all around and separated from underlying cervix. Care should be taken not to be too close to bladder or rectum during dissection. Bladder and pouch of doughlas peritoneum is incised and uterus/cervix delivered depending on the procedure (trachelectomy/radical hysterectomy)

Result(s)* Vaginal is closed with absorbable sutures and specimen sent for final histopathology

Conclusion* Adequate Vaginal margin is a major prognostic factor in cervical cancer. Inadequate or positive margin is associated with recurrence and poor oncological outcomes, hence adjuvant postoperative radiation is indicated in such scenario. Direct visualization and measurement of vaginal to be removed and performing vaginal colpotomy ensures adequate vaginal margin and also prevents the disease being exposed to peritoneal cavity especially in minimal access surgery.

\section{THE UTERINE RADIATION NECROSIS AFTER DEFINITIVE CHEMOIRRADIATION - IMAGING AND CONTROVERSY, A SINGLE CASE REPORT}

M Mikovic*, A Tomasevic, M Radović, D Marjanovic Djoric, I Dedovic Stojakovic, V Plesinac Karapandzic. Institute of Oncology and Radiology of Serbia, Department of Radiotherapy, Belgrade, Serbia

\subsection{6/ijgc-2021-ESG0.55}

Introduction/Background* distinguishing radiation necrosis of uterus and/or cervix from central rest/recurrence after definitive chemoradiation of locally advanced cervical cancer might be challenging, even for experienced clinicians, despite various diagnostic procedures. This is a rare condition and needs to be treated with intensive local care, while central recurrence requires specific oncological treatment with a good prognosis if operable.

Methodology We present a woman, age 40 with severe acute lower abdominal pain, ten months after completing definitive chemoradiation of FIGO stage IIb cervical cancer, with an initially estimated complete treatment regression effect. Histologically, it was large cell nonkeratinizing HG2, NG2 planocellulare invasive carcinoma with a tumor-cervix diameter of $47 \mathrm{~mm}$. Total transcutaneous (TRT) dose of $46 \mathrm{~Gy}$ in 25 fractions was delivered to the whole pelvis (Rapid arc planned), with 5 cycles of weekly Cisplatin-based chemotherapy $\left(40 \mathrm{mg} / \mathrm{m}^{2}\right)$ and 5 intracavitary brachytherapy applications, 1 weekly, with a dose of 7 Gy to reference point A/per application (central tube and two ovoids). After 10 months of complete regression of cancer, clinical exam, ultrasound (US), Positron emission tomography/computed tomography (PET/CT with standardized uptake value, SUV maximum 9.5) and computed tomography (CT) showed an inhomogeneous mass of the cervix, $5 \mathrm{~cm}$ in longitudinal dimension, propagating towards rectum, strongly suspected to recurrence. A biopsy was performed with a result of necrotic inflamed tissue.

Result(s)* Due to the large scale of symptoms of inflammation, specific treatment was not conducted at the time. The patient was treated with supportive therapy, antibiotics, and intensive local care. Five months after the first symptoms, MR showed no signs of disease. The patient is scheduled for further MR control and follow-up.

Conclusion* radiation necrosis must be included in consideration if the result of the biopsy is negative even if most of the diagnostic procedures point towards central recurrent disease.

\section{ESGO QUALITY INDICATORS (QI) IN THE SURGICAL MANAGEMENT OF CERVICAL CANCER. CANARY ISLANDS MATERNAL AND CHILD UNIVERSITY HOSPITAL}

0 Arencibia Sanchez*, AF Rave Ramirez, D González García-Cano, M Laseca Modrego, A Martín Martínez. Complejo Hospitalario Universitario Insular Materno Infantil de Gran Canaria, Gynecologic Oncology, las palmas de gran canaria, Spain

\subsection{6/ijgc-2021-ESGO.56}

Introduction/Background* The objective is to know our degree of compliance with the ESGO 2019 quality indicators in surgical management of cervical cancer

Methodology Retrospective study of patients with cervical cancer who underwent laparoscopic radical hysterectomy in the 
period between 2008-2018.Multiple variables were analyzed related with histopathological study,surgical complications,adyuvant treatments, follow-up and current status of the patients.

Result(s)* 109 radical hysterectomies were performed for cervical cancer during the study period.Average age is 46.5 years (range 25-76 years). Most of the patients $(n=101)$ had stage IB1.The mean tumor size is $1.8 \mathrm{~cm}(0.4-5 \mathrm{~cm})$.In the first 30 days after surgery,3 fistulas were detected.In $99.1 \%$ the margins were free of disease, only one patient presented margin involvement. Two patients had a tumor stage greater than IB1 (1 IB2 and 1 IIA2). The mean number of lymph nodes extracted was 19.8, of those being affected 11.9\% $(n=12)$. $18.3 \%(\mathrm{n}=20)$ received adjuvant treatment with radiotherapy + concomitant chemotherapy,of these 13 were for positive lymph nodes.Therefore, the rate of patients who received adjuvant treatment with $\mathrm{N} 0$ was $8.3 \%$. We have only had one recurrence in less than two years of follow up (1/93).

Conclusion* HUMIC is a reference in gynecological oncology for the province of Las Palmas with trained personnel with exclusive dedication (QI2) and participating in multicenter studies (QI 3).It has a multidisciplinary tumor board where all patients are presented according to recommendations of scientific societies (QI4-5)before and after surgery (QI6-7). We present a $2.7 \%$ urological fistula (QI8 and QI9 <3\%) all of them during learning curve and a patient with BMI of 38 . We reached a $99.1 \%$ rate of free margins of disease (QI10> 97\%).In 2\% we found a staging greater tan IB1 (QI11 $<10 \%$ ). Pelvic lymphadenectomy or SLN (Sentix) was performed at $100 \%$ the patients (QI13> 98\%).8.3\% received adjuvant treatment with N0 (QI15 <15\%). If there is indication, fertility sparing treatment is offered and currently it is performed in our center (QI14 100\%). We had a 2-year recurrence rate of $1 \%$ (QI12 <10\%).

The only indicator we do not reach is the number of cases (minimum QI1 of 15), since our mean is 10 radical hysterectomies per year.Nevertheless, last year we performed 15 surgeries,wich,given our geographical location,we think it allows us to continue as a Reference Center

\section{FERTILITY-SPARING RADICAL TRACHELECTOMY FOR EARLY STAGE CERVICAL CANCER: 12 CASES SERIES AND LITERATURE REVIEW}

N Doan*, T Nguyen, L Ta, L Luong. Oncology Hospital at Ho Chi Minh city, Gynecology Surgery, Ho Chi Minh, Viet Nam

10.1136/ijgc-2021-ESG0.57

Introduction/Background* Radical trachelectomy is an alternative treatment for preserving fertility in selected patients with early stage cervical cancer. The purpose of this report is to describe our technique of abdominal radical trachelectomy and review the current literature on this procedure

Methodology We reported 12 cases of radical trachelectomy with pelvic lymphadenectomy in The Oncology Hospital of Ho Chi Minh city between 7/2018 and 9/2020.

Result(s)* The characteristics of the 12 adult patients who underwent radical trachelectomy included stage IB1 disease in all cases, a mean age of 31 years (range, 29-41), and a median estimated blood loss of $100 \mathrm{ml}$ (range, 70-150). Among of them, one case was performed by laparoscopic approach. No one need adjuvant treatment after sugery and all patients resumed normal menstruation postoperatively. All patients remain disease-free at the time of this report. The only remaining uterine blood supply in these patients are the utero-ovarian vessels. There were one postoperative complication. It was one case of cervical stenosis. Transurethral Foley catheters were removed in all cases at postoperative days $02-04$.

Conclusion* Radical trachelectomy with pelvic lymphadenectomy is a feasible operation for selected women with early stage cervical cancer who desire to preserve reproductive function. Menstruation and reproductive function may be preserved after bilateral uterine vessel ligation.

\section{CERVICAL CANCER APPLICATION (CER-CAP): A NEW APP FOR ESTIMATION OF THE LYMPH NODAL RISK INVASION IN PATIENTS WITH EARLY-STAGE CERVICAL CANCER}

1;2B Guani*, ${ }^{3} \mathrm{~T}$ Gaillard, ${ }^{4} \mathrm{LA}$ Teo-Fortin, 'V Balaya, ${ }^{5} \mathrm{M}$ Plante, ${ }^{3} \mathrm{X}$ Paoletti, ${ }^{3} \mathrm{~F}$ Lécuru, 1;2p Mathevet. 'Lausanne University Hospital, Lausanne, Switzerland; ' ${ }^{2}$ University of Lausanne, Lausanne, Switzerland; ${ }^{3}$ Institut Curie Hospital, Paris, France; ${ }^{4}$ Laval University, Québec, Canada; ${ }^{5}$ Hôtel-Dieu de Québec, Québec, Canada

\subsection{6/ijgc-2021-ESG0.58}

Introduction/Background* Lymph node status is a major prognostic factor in early-stage cervical cancer. According to the International Federation of Gynecology and Obstetrics (FIGO) 2018 classification, the presence of metastatic lymph node involvement, including the presence of macrometastasis (MAC) or micrometastasis (MIC), is classified as stage IIIC. The recommended treatment is a combination of chemoradiation without complete surgery. Assigning initial staging of patients is therefore essential for the therapeutic management.

Methodology We performed a secondary analysis of data from two prospective multicenter trials assessing the role of the sentinel node in the surgical management of cervical cancer (SENTICOL 1 and 2 pooled together in the training dataset). The histological risk factors were included in a multivariate logistic regression model in order to determine the most suitable prediction model. An internal validation of the chosen prediction model was then carried out by a cross validation of the 'leave one out cross validation' type. The prediction model implemented into an interactive online application of the 'Shinyapp' type. Finally, an external validation was performed with a retrospective cohort of L'Hôtel-Dieu de Québec in Canada.

Result(s)* Three hundred twenty-one patients participating in Senticol 1 and 2 were included in our training analysis. Among these patients, 280 did not present lymph node invasion (87.2\%), 13 presented ITC (4\%), 11 presented MIC (3.4\%) and 17 MAC (5.3\%). Tumor size, presence of lymphvascular space invasion and stromal invasion were included in the prediction model. The Receiver Operating Characteristic (ROC) Curve from this model had an area under the curve (AUC) of 0.79 (95\% CI [0.69 - 0.90]). The AUC ROC curve from the cross validation was 0.63 . The external validation on the Canadian cohort confirmed a good discrimination of the model with AUC ROC of 0.83 .

Conclusion* This study is the first study of a prediction score for lymph node involvement in early-stage cervical cancer that includes internal and external validation of a prediction score for lymph node involvement in early-stage cervical cancer. The web application is a simple, practical, and modern method of using this prediction score in clinical management. 